\title{
Management of bilateral malignant ovarian germ cell tumors: Experience of a single institute
}

\author{
TING ZHAO $^{1 *}$, YAN LIU ${ }^{1 *}$, HONGYUAN JIANG ${ }^{1}$, HAO ZHANG $^{2}$ and YUAN LU ${ }^{1}$ \\ Departments of ${ }^{1}$ Gynecology and ${ }^{2}$ Pathology, Obstetrics and Gynecology Hospital, \\ Fudan University, Shanghai 200011, P.R. China
}

Received September 30, 2015; Accepted April 25, 2016

DOI: $10.3892 / \mathrm{mco} .2016 .915$

\begin{abstract}
Bilateral malignant ovarian germ cell tumors (MOGCTs) are rare. Determination of the optimal treatment modalities is crucial, as these malignancies mainly affect girls and young women who may wish to preserve their fertility. In order to review the prevalence, clinical characteristics, treatment and outcome of bilateral MOGCTs, we performed a retrospective review of patients who were diagnosed with bilateral MOGCTs and underwent primary surgery at the Obstetrics and Gynecology Hospital of Fudan University (Shanghai, China) between January, 2001 and December, 2014. Of the 130 patients investigated, 8 were diagnosed with bilateral disease, most of whom were International Federation of Gynecology and Obstetrics stage I. There was no significant difference in overall and disease-free survival between patients with unilateral and those with bilateral disease. Cases with dysgerminoma, dysgerminoma coexisting with gonadoblastoma, yolk sac tumor and ovarian primary choriocarcinoma were included in this study. Fertility was spared in 2 patients ( 1 with dysgerminoma and 1 with ovarian primary choriocarcinoma). The patient with ovarian choriocarcinoma experienced relapse and was finally salvaged by radical surgery and adjuvant chemotherapy. According to our results and the published data, patients affected by bilateral MOGCTs have a satisfactory prognosis. The treatment modalities largely depend on the histological type of the tumor. Fertility-sparing surgery may be safe for patients affected by dysgerminoma, but should be considered with caution in patients with ovarian primary choriocarcinoma.
\end{abstract}

Correspondence to: Dr Yuan Lu, Department of Gynecology, Obstetrics and Gynecology Hospital, Fudan University, 419 Fangxie Road, Shanghai 200011, P.R. China

E-mail: pipiluyuan@163.com

*Contributed equally

Key words: malignant ovarian germ cell tumors, bilateral, management

\section{Introduction}

Malignant ovarian germ cell tumors (MOGCTs) are rare, accounting for $\sim 5 \%$ of all ovarian malignancies (1). These tumors mainly affect girls and young women and are usually diagnosed at early stages. Bilateral involvement in MOGCT patients is very rare, with a reported prevalence of $4.3-6.9 \%(2,3)$. It is crucial to carefully consider the treatment modalities, as the affected patients are usually young and strongly wish to preserve their fertility. Due to the high sensitivity of this type of tumor to chemotherapy, the National Comprehensive Cancer Network of the United States has unequivocally suggested in their guidelines (version 2.2015) that fertility-sparing surgery should be considered, even for patients with advanced-stage disease (4). However, for cases with bilateral involvement, preserving fertility may be associated with increased risk, as cystectomy increases the possibility of residual lesions. Due to the rarity of these tumors, published related articles in the English literature are sparse. An Italian study assessing multicenter data over 20 years aimed to investigate this subject, but only found 8 patients with bilateral involvement, in 4 of whom fertility was preserved (5).

The histological heterogeneity of MOGCTs adds to the complexity of their management. MOGCTs may be divided into dysgerminomas and non-dysgerminomas, of which the most common types are yolk sac tumor, immature teratoma and mixed germ cell tumor. Embryonal carcinoma, non-gestational choriocarcinoma and polyembryoma are rare (4). The grade of malignancy differs between various histological types. The reported 5-year survival rates are also different: For bilateral dysgerminoma, immature terotoma and mixed germ cell tumor, the reported survival rates were 96, 94 and $87 \%$, respectively (2).

Due to the scarcity of bilateral MOGCTs and the varied histological types, it may be difficult to reach a conclusion on whether conservative surgery (cystectomy) increases the risks in these patients. The Obstetrics and Gynecology Hospital of Fudan University (Shanghai, China) is one of China's biggest tertiary referral centers. The aim of this study was to retrospectively analyze the prevalence, clinical characteristics, management and outcome of patients affected by bilateral MOGCTs in this hospital, in order to accumulate more information on this subject. We also conducted a review of the available literature to help elucidate this issue. 


\section{Patients and methods}

Patient selection criteria. Patients meeting the following criteria were included in the study: i) Histologically proven MOGCT; ii) primary surgery performed at the Obstetrics and Gynecology Hospital of Fudan University between January, 2001 and December, 2014. A total of 130 patients fitting the requirements were identified, of whom 8 exhibited bilateral involvement. Patient data, including age at diagnosis, presenting complaint, ultrasonography (USG) findings, serum tumor markers, surgical details, stage and histological type of tumor, adjuvant chemotherapy and follow-up data, including recurrence or death, were retrospectively collected from the hospital medical records. The study protocol was approved by the Institutional Review Board of the Obstetrics and Gynecology Hospital of Fudan University. Written informed consent was acquired from the patients.

Histological classification and staging. The histological diagnosis was based on the International Classification of Diseases for Oncology, 3rd edition, of the World Health Organization (6). Staging was performed according to the International Federation of Gynecology and Obstetrics (FIGO) classification of ovarian tumors (7). The complete staging procedure included the following steps: i) Collection of ascitic fluid or washings of the peritoneal cavity; ii) careful examination of the peritoneum, biopsy and excision of any nodules; iii) omentectomy; and iv) sampling or excision of the pelvic lymph nodes. Fertility-sparing surgery is defined as the preservation of the uterus and at least part of a unilateral ovary, whereas radical surgery is defined as bilateral salpingo-oophorectomy (BSO), with or without total hysterectomy.

Statistical analysis. Statistical analysis was performed with SPSS software, version 16.0 (SPSS Inc., Chicago, IL, USA). Overall survival (OS) was defined as the time interval from the date of primary surgery to death or last visit (months). Disease-free survival (DFS) was defined as the time interval from the date of primary surgery to recurrence or last disease-free visit (months). The Kaplan-Meier method was used to calculate survival and comparisons between survival rates were performed with the $\log$-rank test. P-values $<0.05$ were considered to indicate statistically significant differences.

\section{Results}

Patient characteristics. Between January, 2001 and December, 2014, a total of 130 patients were pathogically diagnosed with MOGCTs and underwent primary surgery at our hospital. The 5-year OS and DFS of all 130 patients were 96.3 and $90.5 \%$, respectively. Eight $(6.2 \%)$ of the patients exhibited bilateral involvement. The demographic and clinicopathological characteristics are listed in Table I. The univariate analysis demonstrated that there was no significant difference in OS and DFS between the unilateral and bilateral groups ( $\mathrm{P}=0.639$ and 0.563 , respectively). The survival curves are presented in Fig. 1.

The median age of the patients was 18 years (range, 17-24 years). A total of 5 patients were admitted to the hospital complaining of a pelvic mass, 1 of irregular vaginal bleeding, and the remaining 2 patients of absence of menarche at the age of 17 years. One patient also complained of frequent urination as an associated symptom. All the patients were nulliparous.

Diagnosis. Two patients presented with bilateral congenital gonadal dysgenesis. The mass in the remaining 6 patients was sized $4.7-30 \mathrm{~cm}$. The tumor size of the bilateral ovaries differed significantly in the same patient, with only one side presenting with an obviously enlarged or giant ovary, while the other ovary was only mildly or moderately enlarged. One patient was diagnosed with FIGO stage IIIC disease, whereas the remaining patients were all FIGO stage IB or IC. Four patients were diagnosed with dysgerminoma, 2 with dysgerminoma coexisting with gonadoblastoma, and the remaining 2 with yolk sac tumor or ovarian primary choriocarcinoma.

Treatment. Laparotomy was performed in 5 and laparoscopy in 3 patients. Four patients underwent complete staging procedures. Fertility was spared in 2 patients (details listed in Table I). All the patients received postoperative chemotherapy, apart from 1 patient diagnosed with dysgerminoma coexisting with gonadoblastoma. The chemotherapy regimens were bleomycin, etoposide and cisplatin (BEP) in 4 patients; cisplatin, vincristine and bleomycin (PVB) in 2 patients and carboplatin, dactinomycin and 5-fluorouracil (CDF) in 1 patient. The number of cycles ranged from 1 to 8 .

Follow-up. During the median follow-up of 83 months (range, 11-429 months), all the patients remained alive. Only 1 patient with bilateral ovarian primary choriocarcinoma experienced recurrence. The patient was a 24-year-old woman who complained of irregular vaginal bleeding for $\sim 1$ month, with a serum $\beta$-human chorionic gonadotropin ( $\beta$-HCG) level elevated to $168.67 \mathrm{IU} / \mathrm{l}$ (normal, <2.7 IU/1). The patient was first diagnosed with ectopic pregnancy and a laparoscopic examination was implemented. A 5-cm cyst was found in the left ovary and a minor break was present in the right ovary which was bleeding, and was considered to be a corpus luteum. The left ovarian cyst was excised and the corpus luteum-like right ovarian lesion was also stripped. On histological examination, both lesions were proven to be primary choriocarcinomas. Following surgery, the CDF regimen was administered for 6 cycles: Carboplatin i.p. on day 1, followed by dactinomycin and 5-fluorouracil i.v. on days 2-6. The $\beta$-HCG level returned to normal, but increased again before the fifth cycle. After the sixth cycle, a second laparotomy was performed, including $\mathrm{BSO}$, hysterectomy, omentectomy, pelvic lymph node dissection and appendectomy. Subsequently, 1 cycle of the CDF regimen and 7 cycles of etoposide, methotrexate, actomycin $\mathrm{D}$, cyclophosphamide and vincristine (EMA-CO) were administered. The $\beta$-HCG returned to normal after the fourth cycle of EMA-CO and did not increase again.

\section{Discussion}

Bilateral MOGCTs are rare, with a reported prevalence of 4.3-6.9\% among MOGCT patients. In the present study, 6.2\% of our MOGCT patients exhibited bilateral involvement, which was consistent with previously reported data. The most common histological type in our series was dysgerminoma, 


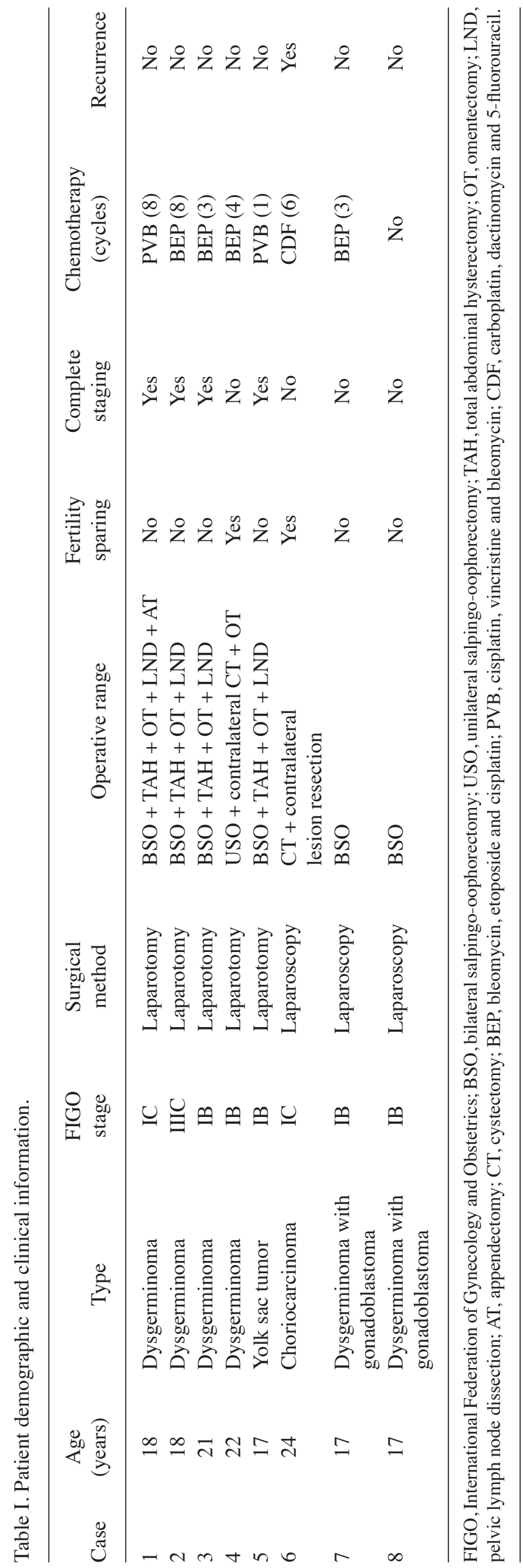

which was also consistent with the published data (5). MOGCTs coexisting with gonadoblastoma in both ovaries are also common, whereas bilateral ovarian yok sac tumor, immature teratoma, embryonal carcinoma and choriocarcinoma are sporadic. The prognosis of bilateral MOGCTs is satisfactory, with no significant difference in OS and DFS between unilateral and bilateral tumors. This result is consistent with a previous study investigating patients identified from the Surveillance, Epidemiology, and End Results (SEER) program, which is the national cancer registry of the United States; the study involved 1,529 patients affected by MOGCTs over a period of 19 years and demonstrated that bilaterality was not an independent predictor of survival (2).

As regards to the treatment of bilateral MOGCTs, we should first emphasize the importance of USG evaluation prior to surgery and careful exploration of bilateral adnexa during surgery, prior to the excision of any tissue. It is particularly important as a sizeable ovarian mass may occupy most of the pelvic cavity, making it difficult to evaluate the contralateral ovary. When bilateral MOGCT is confirmed during surgery, the treatment modalities should be based on the histological type to a certain extent, according to the published literature and our results.

Dysgerminoma is the female analogue of seminoma and the most common type of MOGCT (8). The majority (75\%) of the patients are diagnosed as FIGO stage I and the 5-year survival of the affected patients is $95 \%$ (8). Adjuvant chemotherapy is the only independent prognostic factor for longer DFS and is considered essential for all patients, apart from those with stage IA disease (8).

Bilateral ovarian involvement occurs in $10-15 \%$ of dysgerminoma patients (8). The 5-year survival rate for bilateral dysgerminoma is reported to be $96 \%$, and bilaterality was not found to be an independent prognostic predictor of survival (3). Thus, treatment modalities should be based on those for unilateral disease. It was suggested that, if normal ovarian tissue can be identified, unilateral salpingo-oophorectomy plus contralateral cystectomy or bilateral cystectomy should be taken into consideration (9). However, if both ovaries are almost replaced by neoplastic tissue, attempting to preserve even a small part of the ovary is very likely to result in residual disease. Nevertheless, advanced-stage patients with residual lesions treated with chemotherapy had a satisfactory prognosis (10). In an Italian study, the authors suggested only dysgerminoma histology should be considered for fertility-preserving surgery if residual lesions cannot be avoided (5). Vicus et al reported the case of a 17-year-old patient with bilateral dysgerminoma (FIGO stage IB), who was left with a residual lesion in an attempt to spare fertility. After receiving chemotherapy, the patient had remained relapse-free for 5 years at the time of publication of that case (11). Careful surveillance, including imaging tests and measurement of serum biomarkers, such as carbohydrate antigen-125, is emphasized. Even if both ovaries are destroyed by the tumors, preservation of the uterus for future in vitro fertilization-embryo transfer (IVF-ET) using a donated egg may also be a reasonable choice. In our series, 1 of the 4 bilateral dysgerminoma patients received fertility-sparing surgery; her menstrual cycles remained regular after 4 cycles of BEP regimen and no recurrence was detected during the 102-month follow-up. 

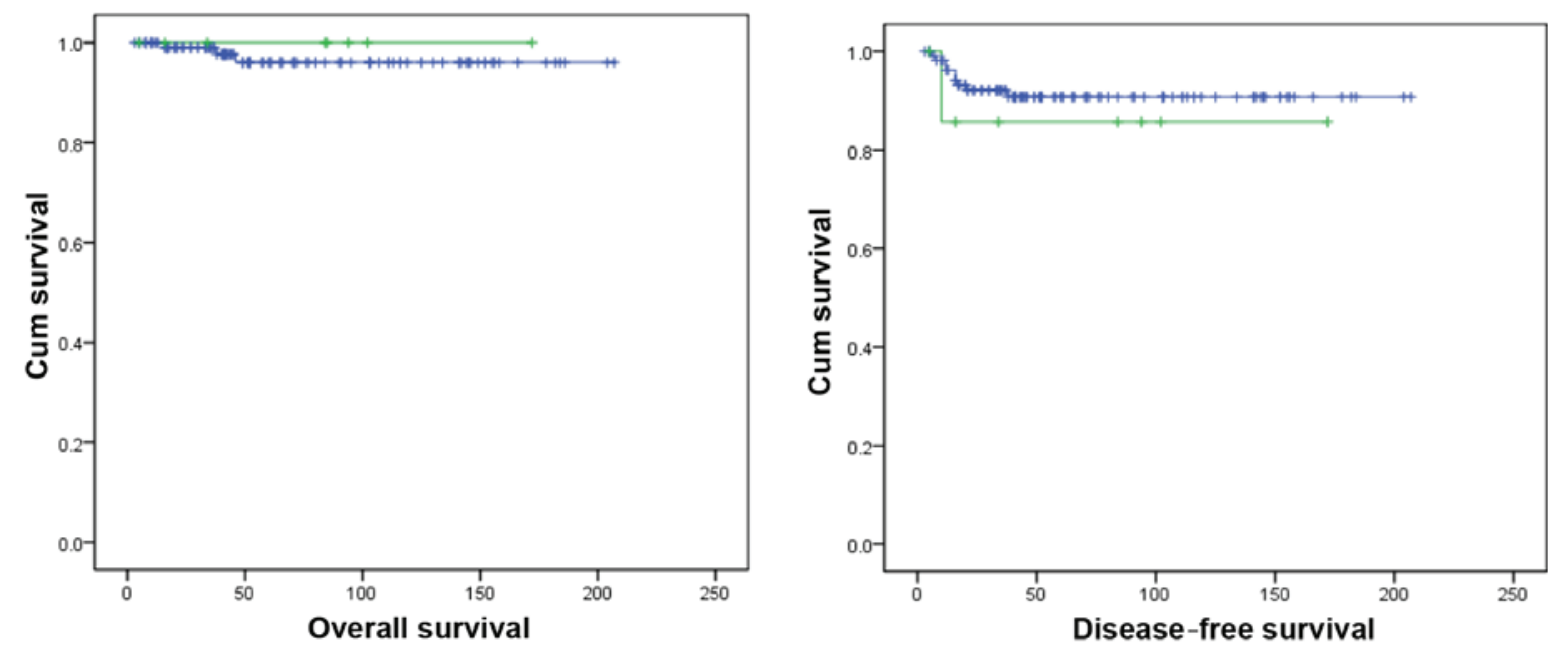

Figure 1. Survival curves of patients affected by unilateral and bilateral malignant ovarian germ cell tumors. Cum, cumulative; 0: unilateral; 1: bilateral.

MOGCTs coexisting with gonadoblastoma account for $10 \%$ of all MOGCTs (12). The most common complaint of the affected patients is absence of menarche. USG examination reveals gonadal congenital dysgenesis, and chromosome examination usually reveals the 46,XY karyotype. Prophylactic bilateral gonadectomy is recommended due to the increased risk of malignant transformation (13), relating to aberrant $\mathrm{Y}$ chromosome gene mutation (14) and microsatellite instability (15). We consider laparoscopy to be safe for such patients. There is no consensus regarding postoperative chemotherapy, as gonadoblastoma, which is a benign neoplasm, usually overgrows other constituents. Hormone therapy may be selected after surgery and successful pregnancy was reported to be achieved in a 46,XY karyotype patient using IVF-ET with a donated egg (16).

Yolk sac tumors are highly aggressive malignancies, usually characterized by early intra-abdominal dissemination and metastasis (17). The reported 5-year survival rates for stage I, II, III and IV disease were $95,75,30$ and $25 \%$, respectively (18). In addition to the FIGO stage, it was reported that cytoreductive surgery significantly affected the prognosis (19). Another study also suggested that the presence of residual tumor was a significant prognostic factor of recurrence (17). These results all support that optimal cytoreductive surgery is vital. The reported rates of bilateral involvement ranged from 6 to $8.4 \%(17,19)$. For bilateral yolk sac tumors, fertility-preserving surgery should be performed with caution, as possible residual disease is very likely to compromise the outcome.

Primary ovarian choriocarcinoma is rare, with an estimated incidence of 1 in 389,000,000 women (20). Bilateral choriocarcinoma of ovarian origin is even rarer. This tumor is highly aggressive and the prognosis is usually poor. For bilateral disease, fertility-sparing surgery is not recommended (20). Postoperative chemotherapy is very important, although, compared with gestational choriocarcinoma, primary ovarian tumors are more chemoresistant (21). The BEP regimen is recommended, as primary ovarian choriocarcinoma is considered to be a germ cell tumor differentiating towards trophoblastic structures (20). However, good prognosis may also be achieved with the EMA-CO regimen (22).
Dactinomycin and 5-fluorouracil were reported to exhibit good efficacy when treating primary tubal choriocarcinoma and gestational choriocarcinoma (23). In our case, fertility was spared at first; however, although chemotherapy with dactinomycin and 5-fluorouracil was administered after surgery, the patient experienced relapse. A second radical operation was performed, the EMA-CO regimen was adopted thereafter and the patient was salvaged.

Immature teratoma was not included in our series. However, several cases of patients who were bilaterally affected by immature teratoma and received cystectomy with chemotherapy have been reported; they were all recurrence-free during a median follow-up of 4.7 years (24). Other types, such as bilateral embryonal carcinoma, should be treated with caution, due to the extremely aggressive behavior of this tumor, as the reported survival rate is only $39 \%$ (5).

In summary, we reviewed the data of patients affected by bilateral MOGCTs over a period of 14 years in the largest oncological center of eastern China and reviewed the related literature, to discuss the issue of preserving fertility in patients with bilateral involvement. The major limitation of this study was the small patient sample. However, bilateral MOGCTs are rare and the published studies are also rare, with limited sample sizes (5). Large-scale studies are required in the future to further investigate this issue.

Patients affected by bilateral MOGCTs have an excellent prognosis. It was hypothesized that conservative treatment should always be preferred, as the tumors are highly sensitive to chemotherapy (5). However, according to our experience and the literature data, the selected treatment modalities should largely depend on the histological type of the tumor. Fertility-sparing surgery may be safe for patients affected by dysgerminoma; however, for primary ovarian choriocarcinoma, the decision to preserve fertility may be associated with significant risks.

\section{Acknowledgements}

This study was supported by grant no. 15140903000 from the Shanghai Science and Technology Commission (Y.L.) 
and grant no. 201540224 from the Shanghai Municipal Commission of Health and Family Planning (Y.L.).

\section{References}

1. Smith HO, Berwick M, Verschraegen CF, Wiggins C, Lansing L, Muller CY and Qualls CR: Incidence and survival rates for female malignant germ cell tumors. Obstet Gynecol 107: 1075-1085, 2006.

2. Mahdi H, Kumar S, Seward S, Semaan A, Batchu R, Lockhart D, Tamimi H and Munkarah AR: Prognostic impact of laterality in malignant ovarian germ cell tumors. Int J Gynecol Cancer 21: 257-262, 2011

3. Roychoudhuri R, Putcha V and Møller H: Cancer and laterality: A study of the five major paired organs (UK). Cancer Causes Control 17: 655-662, 2006.

4. Gershenson DM: Management of ovarian germ cell tumors. J Clin Oncol 25: 2938-2943, 2007.

5. Sigismondi C, Scollo P, Ferrandina G, Candiani M, Angioli R, Viganò R, Scarfone G and Mangili G: Management of bilateral malignant ovarian germ cell tumors: A MITO-9 retrospective study. Int J Gynecol Cancer 25: 203-207, 2015.

6. Fritz A, Percy C, Jack A, Shanmugaratnam K, Sobin L, Parkin DM and Whelan S (eds.): International Classification of Diseases for Oncology (ICD-O). 3rd edition. World Health Organization, Geneva, Switzerland, 2013.

7. Mutch DG, and Prat J. 2014 FIGO staging for ovarian, fallopian tube and peritoneal cancer. Gynecol Oncol. 133: 401-404, 2014.

8. A L Husaini H, Soudy H, El Din Darwish A, Ahmed M, Eltigani A, A L Mubarak M, Sabaa AA, Edesa W, A L-Tweigeri T and Al-Badawi IA: Pure dysgerminoma of the ovary: A single institutional experience of 65 patients. Med Oncol 29: 2944-2948, 2012.

9. Brewer M, Gershenson DM, Herzog CE, Mitchell MF, Silva EG and Wharton JT: Outcome and reproductive function after chemotherapy for ovarian dysgerminoma. J Clin Oncol 17 2670-2675, 1999.

10. Dimopoulos MA, Papadopoulou M, Andreopoulou E, Papadimitriou C, Pavlidis N, Aravantinos G, Aspropotamitis A, Anagnostopoulos A, Fountzilas G, Michalas S and Pectacides D: Favorable outcome of ovarian germ cell malignancies treated with cisplatin or carboplatin-based chemotherapy: A Hellenic Cooperative Oncology Group Study. Gynecol Oncol 70: 70-74, 1998.

11. Vicus D, Beiner ME, Klachook S, Le LW, Laframboise S and Mackay H: Pure dysgerminoma of the ovary 35 years on: A single institutional experience. Gynecol Oncol 117: 23-26, 2010.

12. Lin KY, Bryant S, Miller DS, Kehoe SM, Richardson DL and Lea JS: Malignant ovarian germ cell tumor - role of surgical staging and gonadal dysgenesis. Gynecol Oncol 134: 84-89, 2014.
13. Jonson AL, Geller MA and Dickson EL: Gonadal dysgenesis and gynecologic cancer. Obstet Gynecol 116 (Suppl 2): S550-S552, 2010.

14. Canto P, Söderlund D, Reyes E and Méndez JP: Mutations in the desert hedgehog (DHH) gene in patients with 46,XY complete pure gonadal dysgenesis. J Clin Endocrinol Metab 89: 4480-4483, 2004.

15. Funato T, Uehara S, Takahashi M, Kozawa K, Satoh J, Sasaki T and Kaku M: Microsatellite instability in gonadal tumors of XY pure gonadal dysgenesis patients. Int J Gynecol Cancer 12: 192-197, 2002.

16. Bianco S, Agrifoglio V, Mannino F, Cefalù E and Cittadini E: Successful pregnancy in a pure gonadal dysgenesis with karyotype 46,XY patient (Swyer's syndrome) following oocyte donation and hormonal treatment. Acta Eur Fertil 23: 37-38, 1992.

17. Cicin I, Saip P, Guney N, Eralp Y, Ayan I, Kebudi R and Topuz E: Yolk sac tumours of the ovary: Evaluation of clinicopathological features and prognostic factors. Eur J Obstet Gynecol Reprod Biol 146: 210-214, 2009

18. Nawa A, Obata N, Kikkawa F, Kawai M, Nagasaka T, Goto S, Nishimori K and Nakashima N: Prognostic factors of patients with yolk sac tumors of the ovary. Am J Obstet Gynecol 184: 1182-1188, 2001

19. Kurman RJ and Norris HJ: Embryonal carcinoma of the ovary: A clinicopathologic entity distinct from endodermal sinus tumor resembling embryonal carcinoma of the adult testis. Cancer 38: 2420-2433, 1976

20. Heo EJ, Choi CH, Park JM, Lee JW, Bae DS and Kim BG: Primary ovarian choriocarcinoma mimicking ectopic pregnancy. Obstet Gynecol Sci 57: 330-333, 2014.

21. Koo HL, Choi J, Kim KR and Kim JH: Pure non-gestational choriocarcinoma of the ovary diagnosed by DNA polymorphism analysis. Pathol Int 56: 613-616, 2006.

22. Ozturk E, Ugur MG, Cebesoy FB, Aydin A, Sever T and Balat O: Good prognosis for primary ovarian pure nongestational choriocarcinoma using the EMA/CO regime. Eur J Gynaecol Oncol 31: $123-125,2010$.

23. Wan J, Li XM and Gu J: Primary choriocarcinoma of the fallopian tube: A case report and literature review. Eur J Gynaecol Oncol 35: 604-607, 2014

24. Beiner ME, Gotlieb WH, Korach Y, Shrim A, Stockheim D, Segal Y, Fridman E and Ben-Baruch G: Cystectomy for immature teratoma of the ovary. Gynecol Oncol 93: 381-384, 2004. 